Faktor 4 bewirken, während sich experimentell der Faktor 1,3 ergibt.

Beide Abweichungen würden sich auf eine Beeinflussung des horizontalen Thermodiffusionsstromes zurückführen lassen, wobei bei Innenheizung eine Verminderung, bei Außenheizung dagegen eine Verstärkung anzunehmen ist. Der letztere Vorgang entspricht dann gerade dem praktischen Ziel der Untersuchung (vergl. die Einleitung zu Teil I). Eine kinetische Erklärung dieses Effektes soll zurückgestellt werden, bis experimentell bewiesen ist, daß sich die gleichen Resultate auch mit Isotopengemischen ergeben. Die Vorgänge im außen geheizten Rohr dürften dann für die praktische Isotopentrennung von Bedeutung sein. Darüber hinaus ist die elektrische Turbulenzbeeinflussung auch für die allgemeine Strömungslehre von Interesse, so daß auch in dieser Hinsicht von weiteren Versuchen Aufschlüsse erwartet werden können.

Hrn. Prof. Dr. J. Mattauch danke ich bestens für die gastfreundliche Aufnahme im Institut. Hrn. Dr. habil. L. Waldmann bin ich für fördernde Diskussionen zu Dank vérpflichtet.

\title{
Über die Raman-Spektren von Zink-, Cadmium- und Quecksilberdimethyl sowie von Quecksilbermethylhalogeniden
}

\author{
Von Franz Fehér, Wilhelm Kolb ${ }^{1}$ und Lotte Leverenz ${ }^{2}$ \\ Aus dem Anorganisch-chemischen Institut der Universität Göttingen \\ (7. Naturforschg. 2a, 454-457 [1947]; eingegangen am 14. April 1947)
}

\begin{abstract}
Es werden die Raman-Spektren von Zink-, Cadmium- und Quecksilberdimethyl sowie von Quecksilbermethylhalogeniden mitgeteilt, mit den z. Tl. bereits in der Literatur vorhandenen älteren Werten verglichen und systematisch diskutiert. Eine Deutung der Spektren wird versucht. Dabei wird die bei allen Substanzen auftretende Frequenz zwischen 600 und $700 \mathrm{~cm}^{-1}$ einer extrem niedrigen C-H-Deformationsschwingung zugeordnet. Die Spektren sprechen für eine geradlinige Struktur der Metalldialkyle.
\end{abstract}

$\mathrm{D}^{\mathrm{i}}$ ie Raman-Spektren von Zink- und Quecksilberdimethyl wurden bereits von Ven k a tes waran ${ }^{3}$ und $\mathrm{Pai}^{4}$ aufgenommen. Da die mitgeteilten Frequenzwerte jedoch erheblich differieren und im Falle Pais nachweislich falsche Zuordnungen enthalten, untersuchten wir diese Substanzen noch einmal und führten Polarisationsmessungen ${ }^{5}$ durch. Zum Vergleich wurden außerdem die Spektren von Cadmiumdimethyl und von Quecksilbermethylhalogeniden untersucht.

Die belichteten Substanzen wurden sehr sorgfältig dargestellt und gereinigt, um nach Möglichkeit Verunreinigungen auszuschalten, die zum Teil für die verschiedenen Angaben der früheren Autoren

1 W. Kolb, Diplomarbeit Dresden 1939.

2 L. Leverenz, Diplomarbeit Göttingen 1945.

3 S. Venkateswaran, Ind. J. Physics 5, 145 [1930].

${ }_{4}$ N. G. Pai, Proc. Roy. Soc. [London] 149, 29 [1935].

5 Die Polarisationsmessungen sowie die in Tab. 1-4 unter Fehér angegebenen Werte wurden im Sommer $1940 \mathrm{im}$ Physikalischen Institut der Technischen Hochschule Graz gemessen. Hrn. Prof. Dr. K. W. F. Kohlrausch möchte der eine von uns (Fehér) auch an dieser Stelle nochmals herzlichst für seine damalige Gastfreundschaft danken. verantwortlich waren. Die gemessenen Frequenzwerte sind in Gegenüberstellung zu den älteren Beobachtungen in den Tab. 1-4 zusammengestellt.

\begin{tabular}{|c|c|c|c|c|}
\hline $\begin{array}{c}\text { Venkates- } \\
\text { waran }\end{array}$ & $\mathrm{Pai}$ & Fehér & $\varrho$ & $\begin{array}{c}\text { Kolb, } \\
\text { Leverenz }\end{array}$ \\
\hline & $\begin{array}{l}144(2 d) \\
248(1) \\
488(2)\end{array}$ & & & 144 (sw?) \\
\hline $506(10)$ & $505(8)$ & $\begin{array}{l}503(20 b) \\
523(3)\end{array}$ & 0,18 & $503(s s t)$ \\
\hline $617(2 b)$ & $620(4 d)$ & $\begin{array}{c}621(3 b) ; \\
898(00 ?) \\
1013(00)\end{array}$ & 0,800 & $621(m)$ \\
\hline $1165(8)$ & $\begin{array}{l}1159(6 d) \\
1346(0)\end{array}$ & $\begin{array}{l}1157(15) ; \\
1194(0) \\
1242(0) \\
1313(00 ?)\end{array}$ & 0,32 & $\begin{array}{l}1135(s w) \\
1157(s t) \\
1184(s w) \\
1247(s s w)\end{array}$ \\
\hline $1443(0)$ & & $\begin{array}{l}1432(00) \\
1648(00 ?) \\
2835(1 / 2 b)\end{array}$ & & $\begin{array}{l}1388(s w) \\
1453(s s w) \\
2830(s w)\end{array}$ \\
\hline $2883(8 b)$ & $2897(3)$ & $\begin{array}{l}2902(4) ; \\
2952(1 b)\end{array}$ & $\begin{array}{l}0,05 \\
0,61\end{array}$ & $\begin{array}{l}2893(s t) \\
2940(s w)\end{array}$ \\
\hline
\end{tabular}

Tah. 1. $\mathrm{Zn}\left(\mathrm{CH}_{3}\right)_{2}$. 


\begin{tabular}{|c|c|c|}
\hline Fehér & $\varrho$ & $\begin{array}{c}\text { Kolb, } \\
\text { Leverenz }\end{array}$ \\
& & $150(s s w ?)$ \\
$464(20 b) ;$ & 0,29 & $495(s s t b)$ \\
$468(2 ?) ;$ & & \\
$520(1 / 2 b)$ & & $633(m b)$ \\
$642(3 s b) ;$ & 0,77 & $1128(s t b)$ \\
$934(00)$ & & \\
$1129(8 s b) ;$ & 0,32 & \\
$1384(00 b)$ & & \\
$1448(00)$ & & \\
$2103(1 / 2)$ & & \\
$2218(1 / 2)$ & & \\
$2658(00)$ & & $2898(m s t)$ \\
$2691(00)$ & & \\
$2876(3)$ & & \\
$2902(4) ;$ & $p$ & \\
$2963(1 / 2)$ & & \\
\hline
\end{tabular}

Tab. 2. $\mathrm{Cd}\left(\mathrm{CH}_{3}\right)_{2}$.

\begin{tabular}{|c|c|c|c|}
\hline $\mathrm{Pai}$ & Feh ér & $\varrho$ & $\begin{array}{c}\text { Kolb, } \\
\text { Leverenz }\end{array}$ \\
\hline $\begin{array}{l}156(2 d) \\
255(0) \\
515(8) \\
565(\mathrm{II}) \\
700(3) \\
1182(6) \\
1258(\mathrm{I})\end{array}$ & $\begin{array}{l}514(20 b) ; \\
700(3 b) ; \\
1030(0) ; \\
1180(12) ; \\
1392(0) ; \\
1442(0) ; \\
1706(00) ; \\
2341(0) \\
2868(1 / 2) ; \\
2909(6 b) ; \\
2964(\mathrm{I} b) ;\end{array}$ & $\begin{array}{l}0,23 \\
\\
0,81 \\
0,48 \\
0,37 \\
\\
0,65 \\
0,90 \\
0,77\end{array}$ & $\begin{array}{c}155(s s w ?) \\
514(s s t b) \\
698(\mathrm{mb}) \\
1180(\mathrm{st})\end{array}$ \\
\hline
\end{tabular}

Tab. 3. $\mathrm{Hg}\left(\mathrm{CH}_{3}\right)_{2}$.

Beim Zinkdimethyl sind eine ganze Reihe von Frequenzen von mehreren Autoren gefunden worden. Sie gehören daher sicher zum Schwingungsspektrum des $\mathrm{Zn}\left(\mathrm{CH}_{3}\right)_{2}$. $\mathrm{Zu}$ den anderen Linien ist folgendes zu sagen:

a) Die Frequenzen $1013=2 \times 506$ und $1648=503$ +1145 lassen sich als Ober- bzw. Kombinationstöne erklären. b) Die von $\mathrm{Pa}$ i angegebenen Frequenzen 248 und 1346 lassen sich auf falsche Zuordnung zurückführen, da $\mathrm{Pai}$ nachweislich ohne Filter gearbeitet hat. Beide Linien entsprechen der intensiven $\mathrm{C}-\mathrm{H}$-Schwingung 2897, die im ersten Fall durch die Komponente 24516 des violetten Quecksilber-Tripletts, im zweiten durch die ultraviolette Quecksilberlinie 25592 angeregt wurde. Aufnahmen, die wir ohne Filter machten, bestätigten diese Annahme.

c) Das in Graz aufgenommene Spektrum enthält neben der Linie 503 einen deutlichen Trabanten $523 \mathrm{~cm}^{-1}$. Unsere späteren Versuche zeigten, daß derselbe durch eine Verunreinigung mit Methyljodid verursacht ist, das an dieser Stelle seine stärkste Raman-Frequenz besitzt. Präparate, die unter Umgehung von Methyljodid über Quecksilberdimethyl hergestellt wurden, zeigten selbst bei großer Dispersion keine derartige Linie. Demgegenüber läßt sich die Frequenz 1241 des $\mathrm{Zn}\left(\mathrm{CH}_{3}\right)_{2}$, die im Methyljodid ebenfalls verhältnismäßig intensiv auftritt, nicht durch eine Verunreinigung mit dieser Substanz erklären, da diese Linie auch in den über Quecksilberdimethyl dargestellten Präparaten beobachtet wurde. - Die anderen sehr schwachen Linien des $\mathrm{Zn}\left(\mathrm{CH}_{3}\right)_{2}$ sind möglicher weise ebenfalls auf Verunreinigungen zurückzuführen. Jedoch konnten wir die verunreinigenden Stoffe in diesen Fällen noch nicht eindeutig identifizieren.

Beim Cadmiumdimethyl dürften diejenigen Raman-Frequenzen, die bei beiden Versuchsreihen gemessen wurden, dem Schwingungsspektrum des $\mathrm{Cd}\left(\mathrm{CH}_{3}\right)_{2}$ mit großer Wahrscheinlichkeit angehören. Darüber hinaus muß man wohl auch den beiden Frequenzen 1448 und 2876 eine gewisse Realität zusprechen, wie ein Vergleich mit dem Spektrum des $\mathrm{Zn}\left(\mathrm{CH}_{3}\right)_{2}$ zeigt. Die Linie 468 trat als diffuse Verbreiterung der Hauptlinie 464 auf. Die übrigen sehr schwachen Linien dürften wohl auf Verunreinigungen zurückzuführen sein.

\begin{tabular}{|c|c|c|c|c|c|c|}
\hline $\mathrm{X}$ & \multicolumn{6}{|c|}{$\therefore v$ in $\mathrm{cm}^{-1}$} \\
\hline $\mathrm{Cl}$ & $289(1)$ & $554(12)$ & $1186(8)$; & & $2919(7)$; & $3002(1 / 2 b)$ \\
\hline $\mathrm{Br}$ & verd.; & $545(12)$ & $1172(10)$ & $2796(00)$; & $2921(10)$; & $3005(2 b)$ \\
\hline $\bar{J}$ & verd.; & $532(15)$ & $1162(9)$ & & $2914(0)$; & \\
\hline
\end{tabular}

Tab. 4. $\mathrm{CH}_{3} \mathrm{HgX}$. 
Beim Quecksilberdimethyl gehören wiederum die von allen Autoren übereinstimmend angegebenen Frequenzen mit großer Wahrscheinlichkeit zum Schwingungsspektrum des $\mathrm{Hg}\left(\mathrm{CH}_{3}\right)_{2}$. Von den übrigen Linien läßt sich folgendes sagen:

a) Die Frequenzen $1030=2 \times 515,2341=2 \times 1170$ und $1706=514+1192$ lassen sich als Oberbzw. Kombinationstöne deuten.

b) Im Spektrum Pais läßt sich die Linie 255 auf eine falsche Zuordnung zurückführen. Sie entspricht der starken C-H-Frequenz 2909 und ist durch die ultraviolette Linie 25592 angeregt.

c) Die Frequenzen 565, 1258 und 1392 sind höchstwahrscheinlich auf Verunreinigungen zurückzuführen.

Obgleich wir kleinere Differenzen in den Spektren der Metalldialkyle vorläufig noch nicht eindeutig haben klären können, wollen wir versuchen, das bisherige Beobachtungsmaterial näher zu deuten. Besonders interessiert die Zuordnung der bei allen Substanzen beobachteten intensiven Linie zwischen 600 und $700 \mathrm{~cm}^{-1}$, da vermutet werden kann, daß es sich hier um eine außergewöhnlich niedrige $\mathrm{C}-\mathrm{H}$-Deformationsschwingung handelt. Es soll im folgenden untersucht werden, inwieweit diese Deutungsmöglichkeit den Tatsachen entspricht. Dazu betrachten wir zunächst die Möglichkeit, die betreffende Frequenz als antisymmetrische Valenzschwingung eines linearen oder gewinkelten Dreimassenmodells zu erklären. Die letztere Annahme ist mit unseren Polarisationsmessungen innerhalb der Fehlergrenzen verträglich, wenngleich sich bei allen Substanzen ein Q-Wert ergeben hat, der etwas kleiner als $6 / 7$ ist.

Die Deutung der fraglichen Frequenz als antisymmetrische Valenzschwingung eines linearen Dreimassensystems erscheint von vornherein recht unwahrscheinlich, da bei diesem Modell eine derartige Schwingung im Raman-Effekt verboten ist. Eine Durchbrechung der Auswahlregeln ließe sich nur durch Annahme einer Störung zwischen den Molekülen erklären. Auf jeden Fall müßten aber - wie Modellrechnungen zeigen - die symmetrischen und antisymmetrischen Valenzschwingungen sehr viel näher zusammen liegen, als experimentell beobachtet wurde.

Aus dem gleichen Grunde scheidet die Zuordnung als antisymmetrische Valenzschwingung eines gewinkelten Dreimassensystems aus. Auch hier ist die Differenz der Valenzschwingungen bei allen drei Substanzen im Modell bedeutend kleiner als in Wirklichkeit.

Schließlich kann die fragliche Frequenz auch nicht durch Fermi-Resonanz erklärt werden, da sie in diesem Fall den gleichen Polarisationszustand besitzen müßte wie die Linien um $500 \mathrm{~cm}^{-1}$. Unsere Polarisationsmessungen zeigen aber, daß dies bei keiner der drei Substanzen der Fall ist.

Durch diese negativen Zuordnungsversuche war die Vermutung, daß es sich bei der Frequenz zwischen 600 und $700 \mathrm{~cm}^{-1}$ um eine extrem niedrige $\mathrm{C}-\mathrm{H}$-Deformationsschwingung handelt, beträchtlich erhärtet worden. Leider war es aus Mangel an Deuteriumverbindungen nicht möglich,

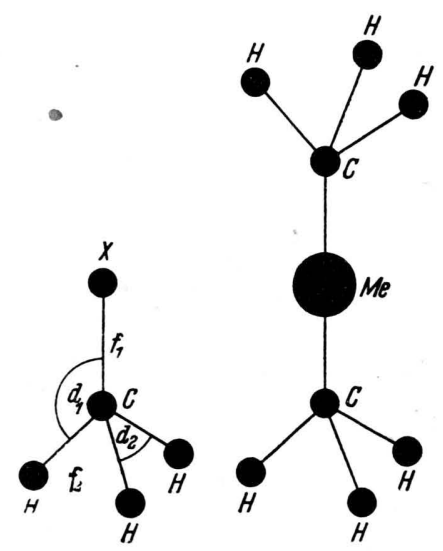

Abb. 1.

diese Zuordnung einwandfrei experimentell $\mathrm{zu}$ entscheiden, so daß wir uns vorläufig auf Modellrechnungen beschränken mußten.

Wir legten diesen Rechnungen das Wagnersche Viermassenmodell ${ }^{6}$ zugrunde. Dasselbe ist in Abb. 1 einem Modell der betrachteten Metalldialkyle gegenübergestellt. Da bei diesen die Kopplung der beiden $\mathrm{CH}_{3}$-Gruppen wegen der sehr viel größeren Masse des Zentralatoms nur sehr gering sein kann, können wir bei der Diskussion der $\mathrm{C}-\mathrm{H}-\mathrm{Frequenzen}$ unsere Moleküle durch das Wagnersche Modell ersetzen. Diese Voraussetzung findet in den Spektren der Substanzen insofern eine Bestätigung, als dieselben bei Vorhandensein einer stärkeren Kopplung sehr viel linienreicher sein müßten.

Wir gingen im einzelnen so vor, daß wir plausible Modellkonstanten in die von Wagner angegebenen Frequenzgleichungen einsetzten und

${ }^{6}$ I. W a gner, Z. physik. Chem. (Abt. B) 40, 36 [1938]. 
zusahen, ob sich eine gewisse Parallelität im allgemeinen Gang der Frequenzen zwischen dem berechneten und dem beobachteten Spektrum ergab. Die Ergebnisse für $\mathrm{Zn}\left(\mathrm{CH}_{3}\right)_{2}$ und $\mathrm{Hg}\left(\mathrm{CH}_{3}\right)_{2}$ zeigen die Tab. 5 und 6.

\begin{tabular}{|l|c|c|}
\hline$\omega$ & berechnet & gemessen \\
\hline$\omega_{4}$ & 503 & 503 \\
$\omega_{5,6}$ & 611 & 621 \\
$\omega_{7}$ & 1196 & 1157 \\
$\omega_{8,9}$ & 1408 & 1453 \\
$\omega_{1}$ & 2858 & 2893 \\
$\omega_{2,3}$ & 2977 & 2940 \\
\hline
\end{tabular}

$f_{1}=1,9 \cdot 10^{5} \mathrm{dyn} / \mathrm{cm} \quad d_{1}=1,3 \cdot 10^{4} \mathrm{dyn} / \mathrm{cm}$ $f_{2}=4,65 \cdot 10^{5} \mathrm{dyn} / \mathrm{cm} \quad d_{2}=4,2 \cdot 10^{4} \mathrm{dyn} / \mathrm{cm}$

Tab. 5. $\mathrm{Zn}\left(\mathrm{CH}_{3}\right)_{2}$.

\begin{tabular}{|l|c|c|}
\hline$\omega$ & berechnet & gemessen \\
\hline$\omega_{4}$ & 507 & 514 \\
$\omega_{5,6}$ & 689 & 698 \\
$\omega_{7}$ & 1252 & 1180 \\
$\omega_{8,9}$ & 1401 & 1433 \\
$\omega_{1}$ & 2875 & 2904 \\
$\omega_{2,3}$ & 2990 & 2967 \\
\hline
\end{tabular}

$f_{1}=2,2 \cdot 10^{5} \mathrm{dyn} / \mathrm{cm} \quad d_{1}=1,6 \cdot 10^{4} \mathrm{dyn} / \mathrm{cm}$

$f_{2}=4,7 \cdot 10^{5} \mathrm{dyn} / \mathrm{cm} \quad d_{2}=4,1 \cdot 10^{4} \mathrm{dyn} / \mathrm{cm}$

Tab. 6. $\mathrm{Hg}\left(\mathrm{CH}_{3}\right)_{2}$.
In beiden Fällen entspricht $\omega_{5,6}$ der fraglichen Frequenz. Die Rechnungen zeigen, daß die gemessenen Spektren im allgemeinen sehr gut mit den nach dem Wagnerschen Modell zu erwartenden übereinstimmen. Insbesondere ist die $\mathrm{C}-\mathrm{H}$-Deformationsschwingung $\omega_{\overline{5}, 6}$ bei den speziellen Massenund Kraftverhältnissen der Metalldialkyle tatsächlich in dem außergewöhnlich niedrigen Frequenzgebiet zwischen 600 und $700 \mathrm{~cm}^{-1}$ zu erwarten.

Zusammenfassend scheint uns danach die $\mathrm{Zu}$ ordnung der bei allen Metalldialkylen zwischen 600 und $700 \mathrm{~cm}^{-1}$ auftretenden Frequenz als abnorm niedrige $\mathrm{C}-\mathrm{H}$-Deformationsschwingung die wahrscheinlichste zu sein, zumal dieselbe auch mit unseren Polarisationsmessungen im Einklang steht $(d p)$. Für $\mathrm{Cd}\left(\mathrm{CH}_{3}\right)_{2}$ wurde die Rechnung nicht besonders durchgeführt, da zu erwarten war, daß es sich in seinem Verhalten in die Reihe der Metalldialkyle zwischen $\mathrm{Zn}\left(\mathrm{CH}_{3}\right)_{2}$ und $\mathrm{Hg}\left(\mathrm{CH}_{3}\right)_{2}$ einreiht.

Die restlichen Frequenzen der Metalldialkyle lassen sich bei allen drei Substanzen am besten bei Annahme einer linearen Kette zuordnen. Für diese Struktur spricht auch der allgemeine Gang der Frequenzen beim Übergang von $\mathrm{Zn}\left(\mathrm{CH}_{3}\right)_{2} \mathrm{zu}$ $\mathrm{Hg}\left(\mathrm{CH}_{3}\right)_{2}{ }^{7}$.

7 Siehe auch K. W. F. Kohlrausch, Raman-Spektren, Hand- und Jahrbuch d. chem. Physik 9, VI, S.183; dort auch kurze Diskussion der Quecksilbermethylhalogenid-Spektren (S. 417). 